\title{
Long-Range Energy-State Maneuvers for Minimum Time to Specified Terminal Conditions ${ }^{1}$
}

\author{
M. G. Parsons, ${ }^{2}$ A. E. Bryson, JR., 3 AND W. C. Hoffman 4 \\ Communicated by A. Miele
}

Dedicated to Professor A. Busemann

\begin{abstract}
Some three-dimensional minimum-time paths to a specified final line (or point), heading, and energy are presented for an example supersonic aircraft. The optimum maneuvers have been determined using the calculus of variations and the energystate approximation. These are compared with suboptimal solutions obtained with the additional assumption that only three discrete values of bank angle $\left(-\phi_{\max }, 0,+\phi_{\max }\right)$ are available. Constraints on thrust, Mach number, angle-of-attack, dynamic pressure, and load factor are included. For ranges long enough that maximum velocity is attained en route, the initial and final arcs can be determined separately, which greatly simplifies the solutions.
\end{abstract}

Key Words. Calculus of variations and applications, flight mechanics, suboptimal control, bounded control problems, chattering phenomena.

1 This research was supported by ONR Contract No. N00014-67-A-0112-0063, Work Unit No. NP 213-085; NASA Grant No. 05-020-007; and AFOSR Contract No. F44620-72-C-001.

2 Assistant Professor, Department of Naval Architecture and Marine Engineering, University of Michigan, Ann Arbor, Michigan.

${ }^{3}$ Professor and Chairman, Department of Aeronautics and Astronautics, Stanford University, Stanford, California; Consultant and Director, Aerospace Systems, Inc., Burlington, Massachusetts.

4 Project Engineer, Aerospace Systems, Inc., Burlington, Massachusetts. 


\section{Nomenclature}

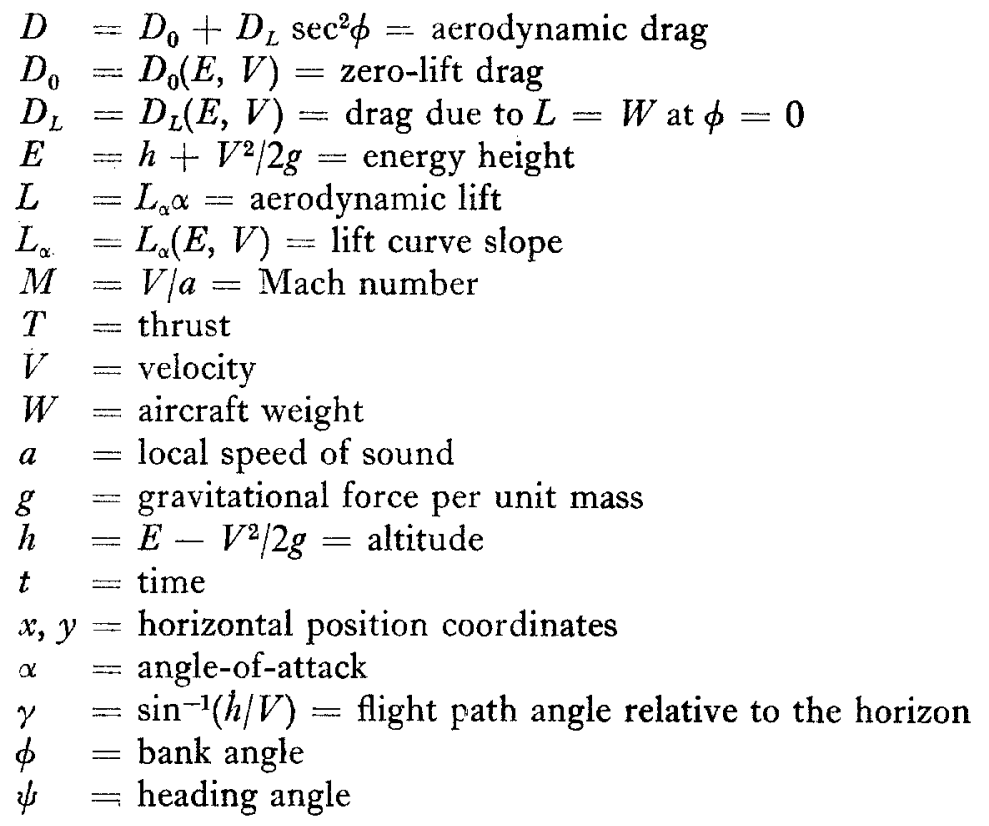

\section{Subscripts}

$$
\begin{aligned}
& f=\text { final value } \\
& \max =\text { maximum value } \\
& 0 \quad=\text { initial value }
\end{aligned}
$$

\section{Introduction}

Use of the energy-state approximation greatly simplifies the determination of optimal flight paths for aircraft with only a small reduction in accuracy (see, e.g., Refs. 1 and 2). For three-dimensional flight paths the energy-state model requires three control variables: thrust $T$, bank angle $\phi$, and altitude $h$. For paths to a line (see Fig. 1a), the model uses only three state variables, energy $E$, heading $\psi$, and distance to the line $x$. For paths to a point (see Fig. 1b), an additional state variable is needed: lateral position perpendicular to the line connecting the initial and final points $y$. The equations of motion and applicable constraints are summarized in Section 2 . 


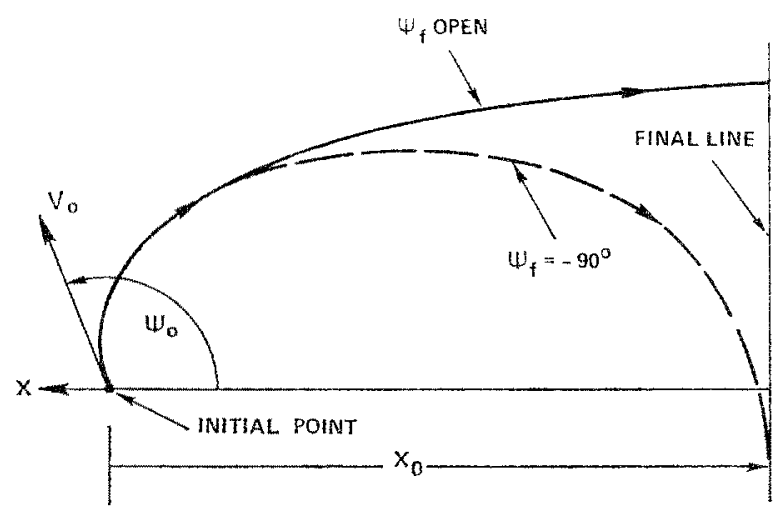

(a) Flight Path to a Line

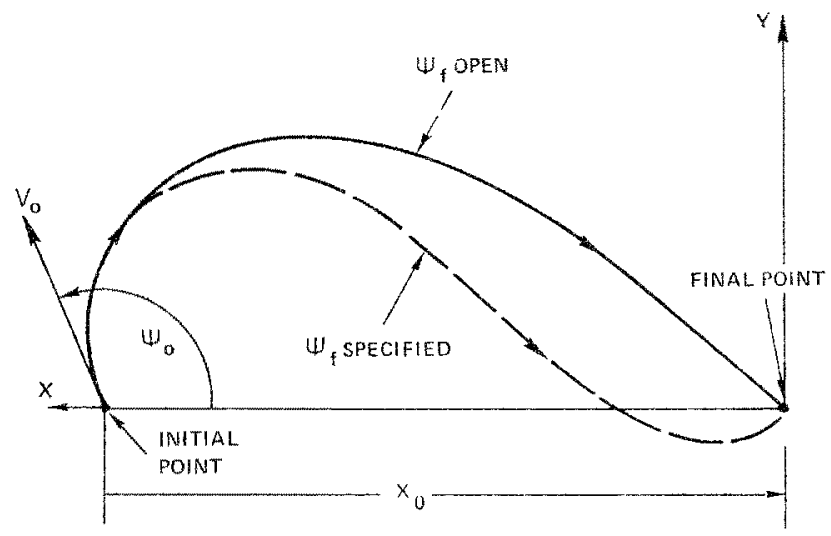

(b) Flight Path to a Point.

Fig. 1. Horizontal projection of minimum-time paths: (a) to a line and (b) to a point.

If the initial range $x_{0}$ is sufficiently large that maximum velocity $V_{\max }$ is reached in transit, the minimum-time path to a line can be separated into two parts (see Fig. 2a). First, the minimum-time path is found to a straight $V_{\max }$ arc perpendicular to the line (i.e., $\psi=0$ ). Second, the minimum-time turn from the $V_{\max }$ arc to the line is determined. The length of the intermediate $V_{\max }$ arc then is obtained such that the sum of the ranges traversed on the three segments equals $x_{0}$.

This separation of arcs greatly simplifies the solution, since the initial-arc problem has only two parameters $\left(E_{0}, \psi_{0}\right)$ and the final-arc 


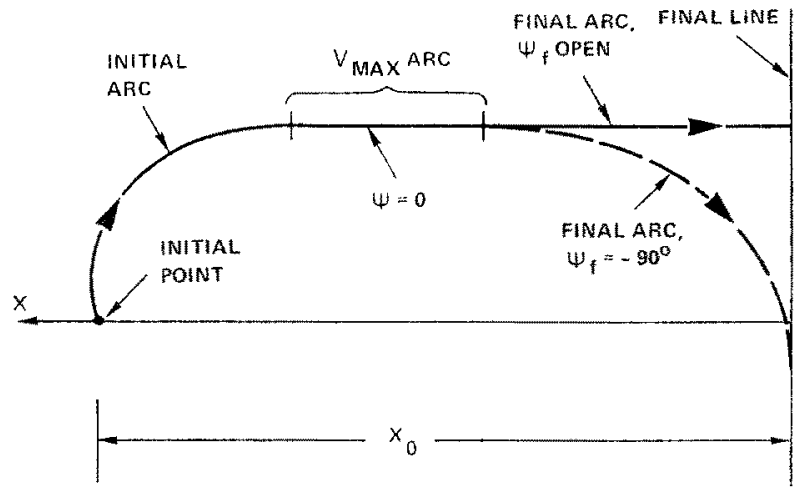

(a) Flight Poth to a Line.

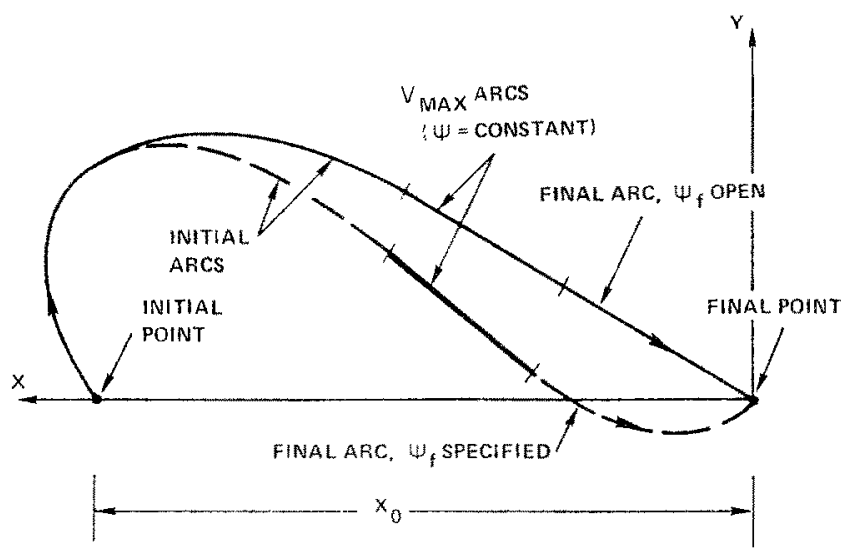

(b) Flight Fath to a Point.

Fig. 2. Horizontal projection of minimum-time paths: (a) to a line and (b) to a point.

problem has only two parameters $\left(E_{f}, \psi_{f}\right)$. Each of these two problems can be solved by generating a one-parameter family of arcs. However, for short ranges (no $V_{\max }$ arc) the problem has five parameters $\left(E_{0}, \psi_{0}\right.$, $x_{0}, E_{f}, \psi_{f}$ ), and the tabulation of solutions is virtually impossible.

The arcs can also be separated, for sufficiently long range, in determining minimum-time paths to a point (see Fig. $2 \mathrm{~b}$ ). However, in addition to the length of the $V_{\max }$ arc, its heading and its intercept distance $\left(x\right.$-coordinate where the $V_{\max }$ arc or its extension intercepts $y=0$ ) must also be determined. This can be done iteratively using the two-parameter families of initial and final arcs mentioned previously. 


\section{Equations of Motion and Constraints}

The equations of motion for three-dimensional flight using the energy-state approximation are (see nomenclature):

$$
\begin{aligned}
\dot{E} & =(V / W)\left(T-D_{0}-D_{L} \sec ^{2} \phi\right), \\
\psi & =(g / V) \tan \phi, \\
\dot{x} & =-V \cos \psi, \\
\dot{y} & =V \sin \psi .
\end{aligned}
$$

The state variables are $E, \psi, x$, and $y$; the three control variables are $T, V$, and $\phi$. The auxiliary variables $h, \alpha$, and $\gamma$ are defined in the nomenclature. These equations assume: (a) $|\alpha| \ll 1$ and $|\gamma| \ll 1$; (b) $V|\dot{\gamma}| \ll g$; (c) instantaneous velocity changes possible at constant $E$ (zoom dives or climbs); (d) $T \ll L_{\alpha}$; and (e) weight change is negligible during the maneuver.

The following constraints are observed:

$$
\begin{gathered}
0 \leqslant T \leqslant T_{\max }(E, V), \\
V_{\min }(E, \phi) \leqslant V \leqslant V_{\max }(E), \\
-\phi_{\max } \leqslant \phi \leqslant+\phi_{\max },
\end{gathered}
$$

where $T_{\max }(E, V)=$ maximum available thrust and $\tan \phi_{\max }=$ maximum permissible turning load factor $(g$ 's $)$. The maximum velocity constraint is imposed by engine and dynamic pressure limits and the constraint that $h \geqslant 0$ (see Fig. 3). The minimum velocity constraint (see Figs. 3 and 7 ) results from specifying a maximum angle-of-attack: ${ }^{5}$

$$
\alpha=W \sec \phi / L_{\alpha}(E, V) \leqslant \alpha_{\max } .
$$

The bank angle constraint assures that the crew are not subjected to excessively high load factors. ${ }^{6}$

\section{Optimality Conditions}

The variational Hamiltonian is given by

$$
H=1+\lambda_{E} \dot{E}+\lambda_{\psi} \psi+\lambda_{y} \dot{x}+\lambda_{y} \dot{y},
$$

\footnotetext{
${ }^{5} \alpha_{\operatorname{mix}}$ was taken as $12^{\circ}$ for our examples.

${ }^{6} \phi_{\max }$ was taken as $\approx 76^{\circ}$ for our examples $\left(\tan \phi_{\max }=4\right)$.
} 


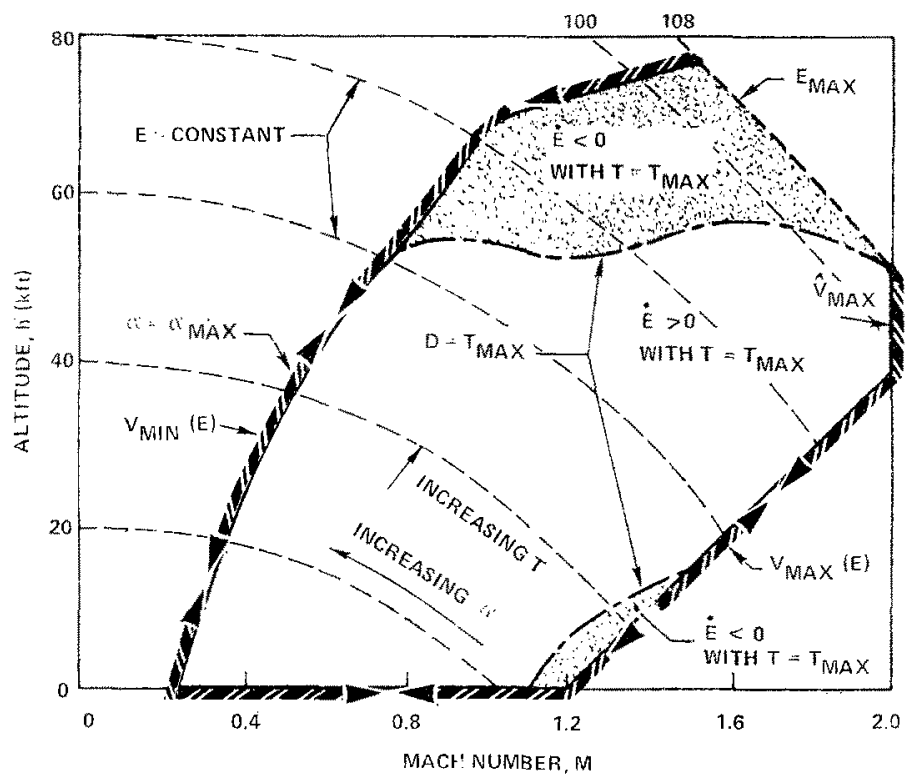

Fig. 3. Aircraft flight envelope with $\phi=0, L \approx W$.

where

$$
\dot{\lambda}_{E}=-\partial H / \partial E, \quad \dot{\lambda}_{\psi}=-\partial H / \partial \psi, \quad \lambda_{x}=0, \quad \dot{\lambda}_{y}=0,
$$

and

$$
T, V, \phi=\arg \min _{T, \forall, \phi} H .
$$

Note that $\partial H / \partial E$ includes terms proportional to $\partial T_{\max } / \partial E, \partial V_{\max } / \partial E$, or $\partial V_{\text {mix }} / \partial E$ if the optimal path is on the $T_{\max }, V_{\max }$, or $V_{\min }$ constraint boundaries, respectively.

Transversality at the final time requires that

$$
H\left(t_{f}\right)=0 \text {. }
$$

Boundary conditions for minimum time to a point are:

$$
\begin{aligned}
& E(0)=E_{0}, \quad \psi(0)=\psi_{0}, \quad x(0)=x_{0}, \quad y(0)=0, \\
& E\left(t_{f}\right)=E_{f}, \quad \lambda_{\psi}\left(t_{f}\right)=0, \quad x\left(t_{f}\right)=0, \quad y\left(t_{f}\right)=0 .
\end{aligned}
$$

Integrals of the optimum path are

$$
H=0, \quad \lambda_{x}=\text { const }, \quad \lambda_{\nu}=\text { const. }
$$




\section{Aircraft Flight Envelope for Zero Bank Angle}

Figure 3 shows the flight envelope of an early F4 (Phantom) airplane at a weight $W$ of $35,000 \mathrm{lb}$ for zero bank angle $(\phi=0)$ and lift $L$ approximately equal to $W$ (see Ref. 3 for thrust, lift, and drag data). The aircraft can move along constant energy contours $(E=$ const) very quickly by zoom dives or climbs (effected by changing angle-of-attack). The highaltitude, low-velocity limit of zoom climbs is the maximum angle-ofattack $\left(x_{\max }\right)$ boundary. Here, we took $\alpha_{\max }=12^{\circ}$. The low-altitude, high-velocity limit of zoom dives is the maximum velocity $\left(V_{\max }\right)$ boundary or the zero altitude $(h=0)$ boundary. $\hat{V}_{\max }$ is the maximum velocity independent of $E$. Also shown are the contours where drag $D$ equals maximum thrust $T_{\max }$. These contours separate regions where $\dot{E}<0$ and $\dot{E}>0$ with $T=T_{\max }$. Energy is changed by adjusting the thrust. ${ }^{7}$ The region where $\dot{E}>0$ with $T=T_{\max }$ is quite large and extends up to $E=E_{\max }=108 \mathrm{kft}$.

\section{Minimum-Time Paths to a Given Long Range: $\psi_{0}=0$, $\psi_{f}$ Unspecified}

Figure 4 shows the altitude-Mach number profile of flight paths that yield minimum time to a given long range when no turning or

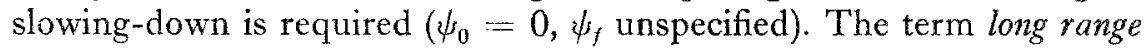
will be used henceforth to indicate that a $V_{\max }$ arc occurs on the path. An example is indicated for $E_{0}=E_{j}=3 \mathrm{kft}$. For other values of $E_{0}$ and $E_{j}$, zoom maneuvers at constant $E$ to the ascent path and from the descent path are used. The ascent $(\dot{E}>0)$ path uses $T=T_{\max }$ but is not the same as the path for maximum $\dot{E}$ (also shown in Fig. 4). The $\psi=-0$ minimum-time path has higher velocity at each energy level than the $\dot{E}_{\max }$ path (see Ref. 1). The descent path is along the $V_{\max }(E)$, $(-\dot{E})_{\text {In:x }}$ boundary using $T=0$ and chatter, i.e., banking rapidly first to $+\phi_{\max }$, then to $-\phi_{\max }$, in order to maximize the drag due to lift $\left(L=W \sec \phi_{\max }\right)$.

Figure 5 shows the altitude-range profile of the flight paths in Fig. 4. For long ranges, the initial and final arcs are determined separately; a $V_{\text {max }}$ cruise arc is inserted between them with appropriate range so that

\footnotetext{
7 Note that, at low energies, the constant energy contours are almost parallel to constant altitude contours, so angle-of-attack primarily controls speed while thrust primarily controls altitude. At high energies, the constant energy contours are almost parallel to constant speed curves, so $\alpha$ primarily controls altitude while $T$ primarily controls speed.
} 


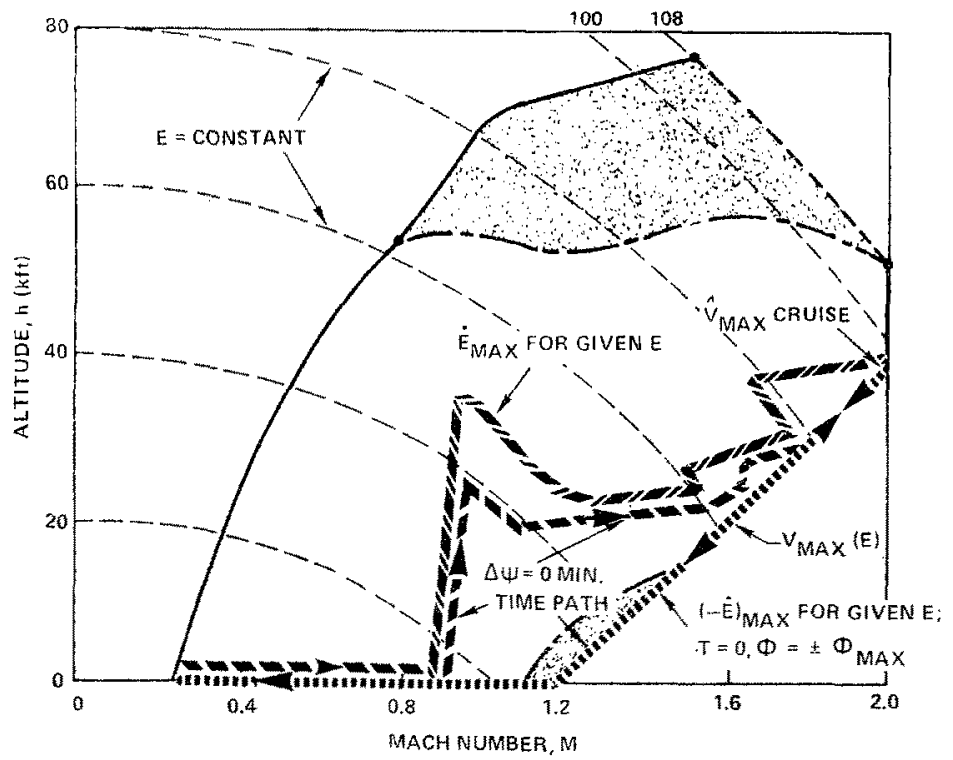

Fig. 4. Altitude-Mach number profile of $\psi=0$ path for minimum time to a given long range.

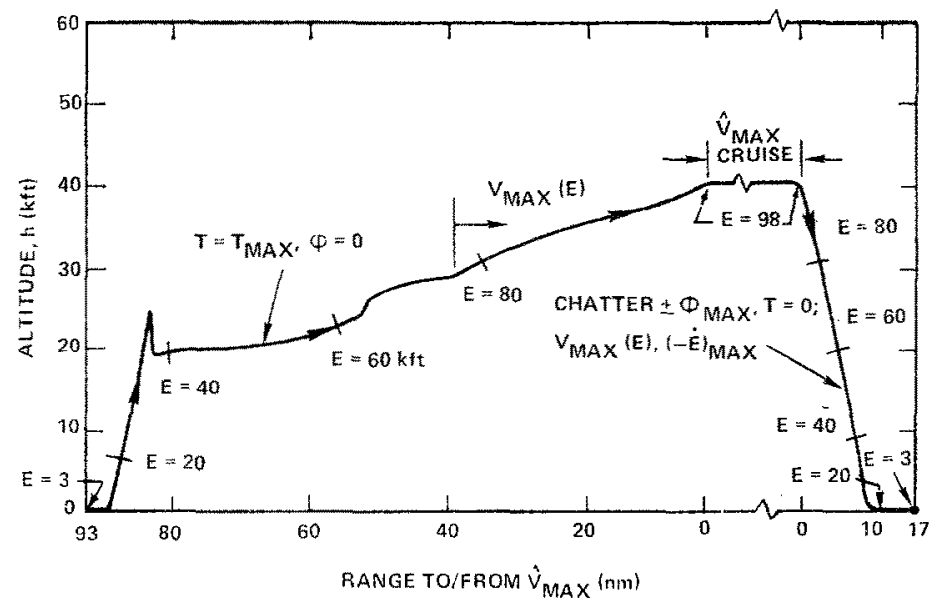

Fig. 5. Altitude-range profile of $\psi=0$ path for minimum time to a given long range. 
the total range is the specified $x_{0}$. For example, with $E_{0}=E_{f}=40 \mathrm{kft}$, any range greater than $88 \mathrm{~nm}$ will include an initial arc of $80 \mathrm{~nm}$ range, a $V_{\max }$ arc, and a final arc of $8 \mathrm{~nm}$ range.

\section{Aircraft Flight Envelope for Maximum Bank Angle Turning}

Figure 6 shows the flight envelope of the same airplane in turning maneuvers at maximum bank angle $\left(\phi=\phi_{\max }\right)$, with $L \cos \phi_{\max }=W$, i.e., the vertical component of lift equal to weight. Here, we took $\tan \phi_{\max }=$ maximum load factor $=4$. The maximum angle-of-attack boundary is also the locus of maximum turn-rate $\psi_{\max }$ and minimum velocity for a given energy $V_{\min }(E)$. The importance of this locus was first pointed out in Ref. 4 where it was called the corner locus. This boundary is now substantially lower than in Fig. 3, as is the contour where $D=T_{\max }$. The intersection of these two contours at $h=20 \mathrm{kft}$, $M=0.75$ is the highest energy where maximum turn rate can be sustained with $T=T_{\max }, \phi=\phi_{\max }, \alpha=\alpha_{\max }$. The maximum steady turn rate occurs at sea level, $M=0.25$, with $T<T_{\max }$, $\phi=\phi_{\max }, \alpha=\alpha_{\max }$. The region where $\dot{E}>0$ with $T=T_{\max }$, $\phi=\phi_{\max }$ is relatively small and occurs in the transonic speed regime below $h=20 \mathrm{kft}$.

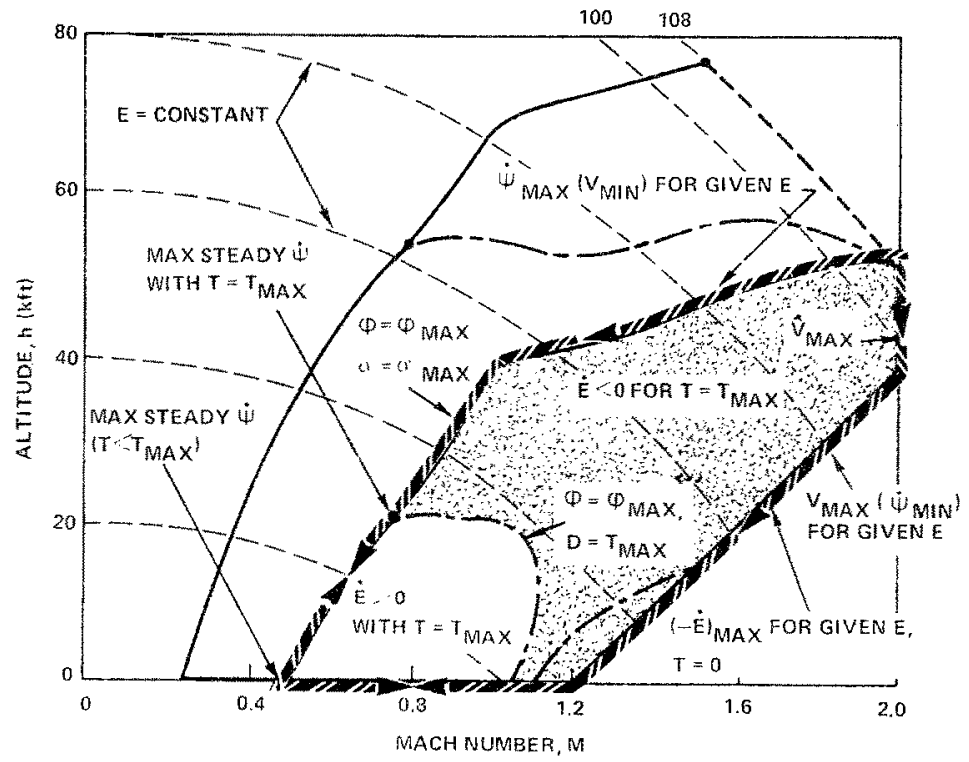

Fig. 6. Aircraft Aight envelope for $\phi=\phi_{\max }, L \cos \phi_{\max }=W$. 


\section{Minimum-Time Paths to a Line (Long Range): $\psi_{0} \neq 0$, $E_{f}$ and $\psi_{f}$ Unspecified}

Figure 7 shows a horizontal projection of a typical minimum-time path to a given line which is far enough away that a $V_{\text {max }}$ cruise arc occurs en route. The case shown is for $E_{0}=43 \mathrm{kft}, \psi_{0}=180^{\circ}$. The continuous bank angle solution (Ref. 5) uses a $\psi_{\max }$ turn for the first $110^{\circ}$ (to $\psi=70^{\circ}$ ), then a gradually decreasing altitude and bank angle to the $\Delta \psi=0$ minimum-time locus of Fig. 4 . Note that $\psi=0$ is not reached exactly until $V=V_{\max }$ at a range of $86 \mathrm{~nm}$. Also shown in Fig. 7 is a suboptimal path with $\phi$ limited to three discrete values $\left(-\phi_{\max }, 0,+\phi_{\operatorname{mix}}\right)$. It consists of a $\psi_{\max }$ turn all the way to $\psi=0$ (which occurs at a range change of $0.5 \mathrm{~nm}$ ), then a zoom dive to the $\Delta \psi=0$ minimum-time locus of Fig. 4 . Both paths reach $V_{\max }$ at substantially the same time and distance $(86 \mathrm{~nm})$. Because of its simplicity, this suboptimal path provides a significant reduction in computational requirements with only a slight increase in flight time. This model appears to be quite similar to the energy-maneuverability model of Boyd (Refs. 6 and 7).

There are four types of minimum-time turns to a given long range where $E_{f}$ and $\psi_{f}$ are unspecified. Figure 8 shows the accelerating turn case where $E_{0} \leftarrow 30 \mathrm{kft}$. For $\psi_{0}=0$, the aircraft zoom dives or climbs and follows the $\Delta \psi=0$ minimum-time path. For $0^{\circ}<\psi_{0} \leqslant 180^{\circ}$, the aircraft zoom dives or climbs to the $\psi_{\max }$ locus where an accelerating turn takes place, followed by a zoom dive to the $\Delta \psi=0$ minimum-time path. Figure 9 shows the constant-velocity turn case where $E_{0}=30 \mathrm{kft}$. Here, the aircraft zoom dives or climbs to the $\dot{\psi}_{\max }$ locus where a constant-velocity turn takes place, followed by a zoom dive to the

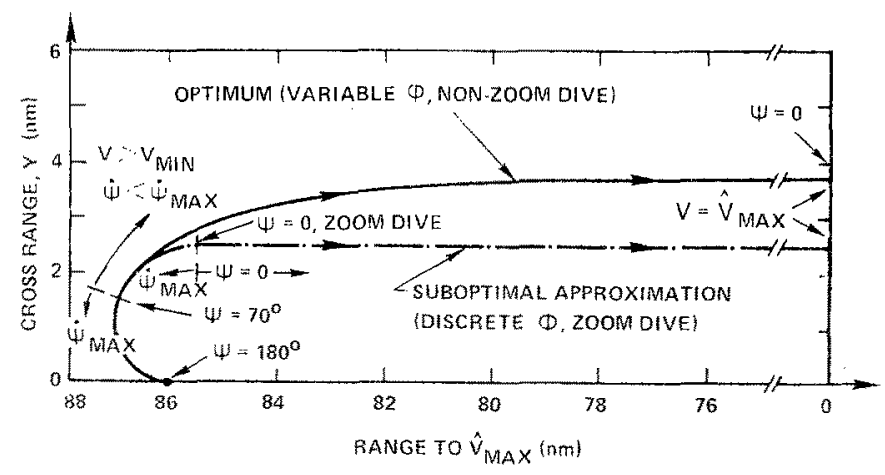

Fig. 7. Horizontal projection of a typical minimum-time turn to a given long range, $E_{0}=43 \mathrm{kft}$. 


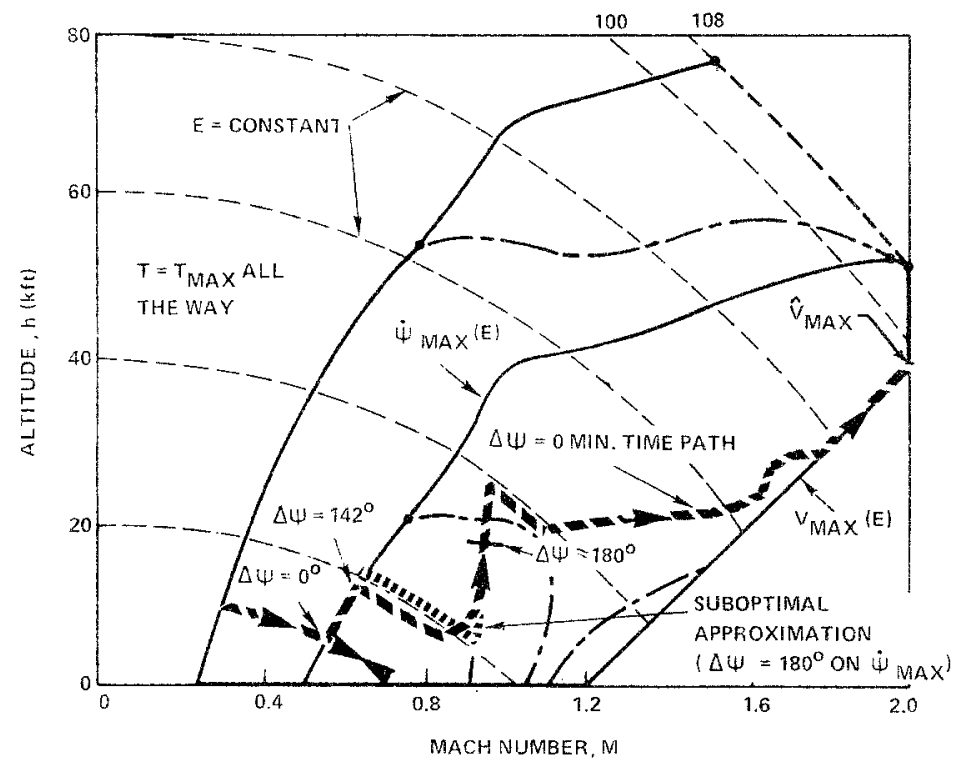

Fig. 8. Minimum-time turns to a given long range $\left(E_{f}, \psi_{f}\right.$ unspecified), $E_{0}<30 \mathrm{kft}$.

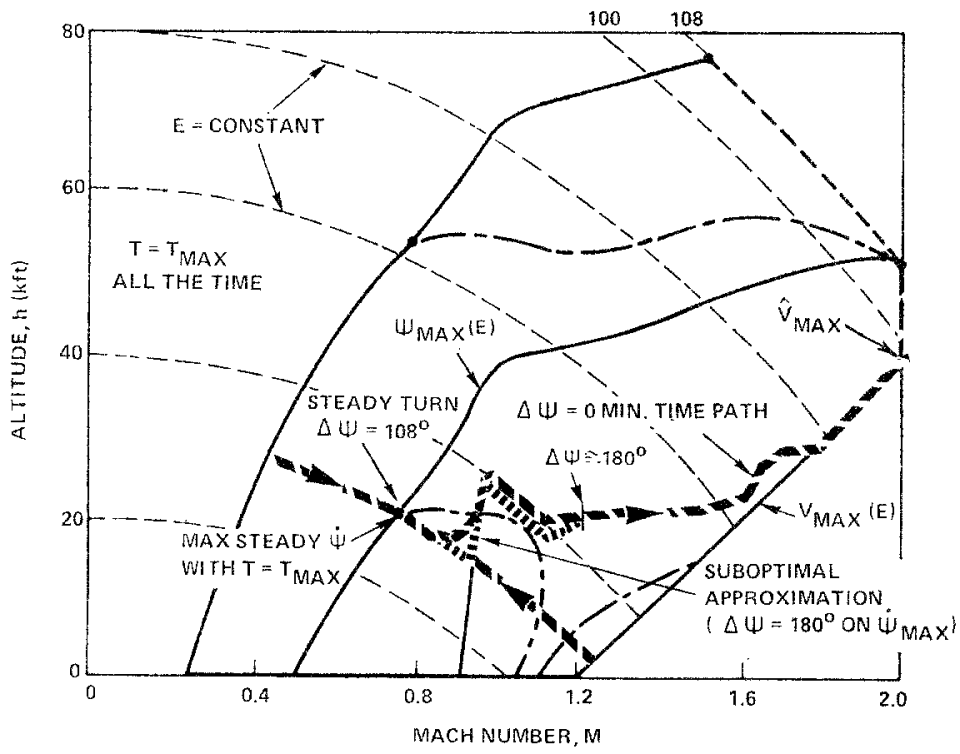

Fig. 9. Minimum-time turns to a given long range $\left(E_{f_{1}} \psi_{f}\right.$ upspecified), $E_{0}=30 \mathrm{kft}$. 


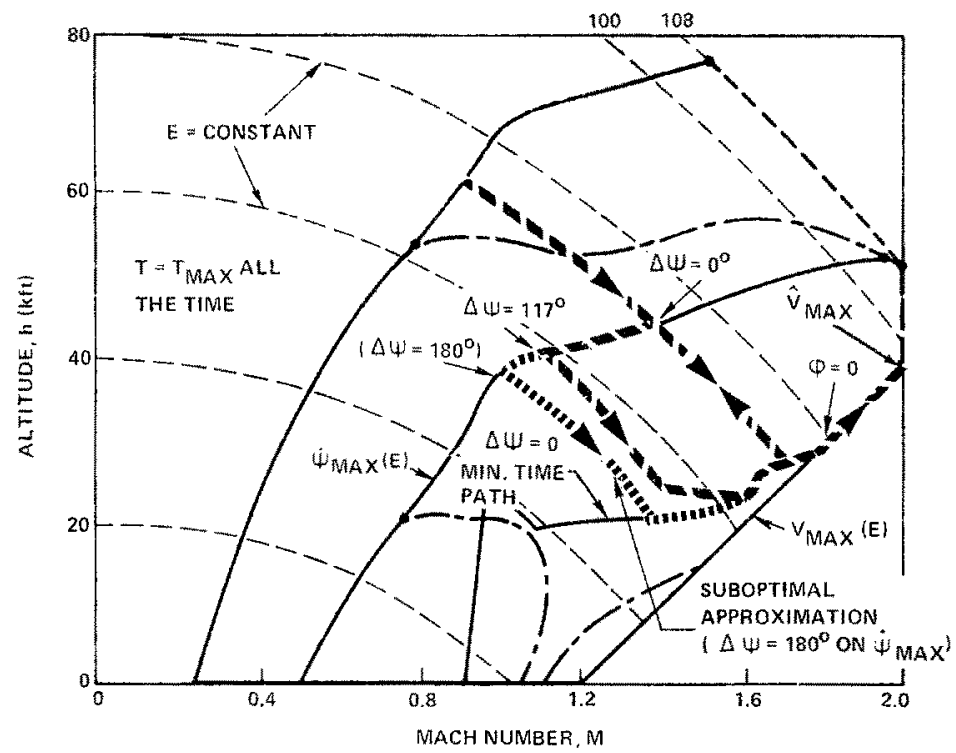

Fig, 10. Minimum-time turns to a given long range $\left(E_{f}, \psi\right.$ unspecified), $30 \mathrm{kft}<E_{0}<93 \mathrm{kft}$.

$\Delta \psi=0$ minimum-time path. Figure 10 shows a decelerating turn case where $30 \mathrm{kft}<E_{0}<93 \mathrm{kft}$. The aircraft zoom dives or climbs to the $\psi_{\max }$ locus where a decelerating turn takes place, followed by a zoom dive to the $\Delta \psi=0$ minimum-time path. The examples of Figs. 8-10 all use $T=T_{\max }$. For $E_{0}>95+\left(180-\psi_{0} / 13\right) \mathrm{kft}$, an initial period of $T=0$ is required. An example of such a case is shown in Fig. 11 for $E_{0}=E_{\max }=108 \mathrm{kft}$ and $\psi_{0}=180^{\circ}$. The aircraft turns about $15^{\circ}$ before $T$ is switched from zero to $T_{\max }$ to complete the turn.

Figure 12 shows all minimum-time turns to a given long range ( $E_{f}, \psi_{f}$ unspecified) with $E_{0}, \psi_{0}$ as parameters. Note that there is a range of possible $E_{f}$ from 98 to $108 \mathrm{kft}$ at $\hat{V}_{\max }$. The suboptimal paths (i.e., those with a $\psi_{\max }$ turn all the way to $\psi=0$, then a zoom dive to the $\Delta \psi=0$ minimum-time path) are also depicted.

\section{Minimum-Time Flight Paths to a Point or Line from $V_{\max }$ Cruise: $E_{f}$ and $\psi_{f}$ Specified}

Figures 13 and 14 show a horizontal projection and an $h$ versus $M$ profile, respectively, of a typical minimum-time path to a point or line from $\hat{V}_{\max }$ cruise. The case shown is for $E_{f}=37 \mathrm{kft}, \Delta \psi=180^{\circ}$. It 


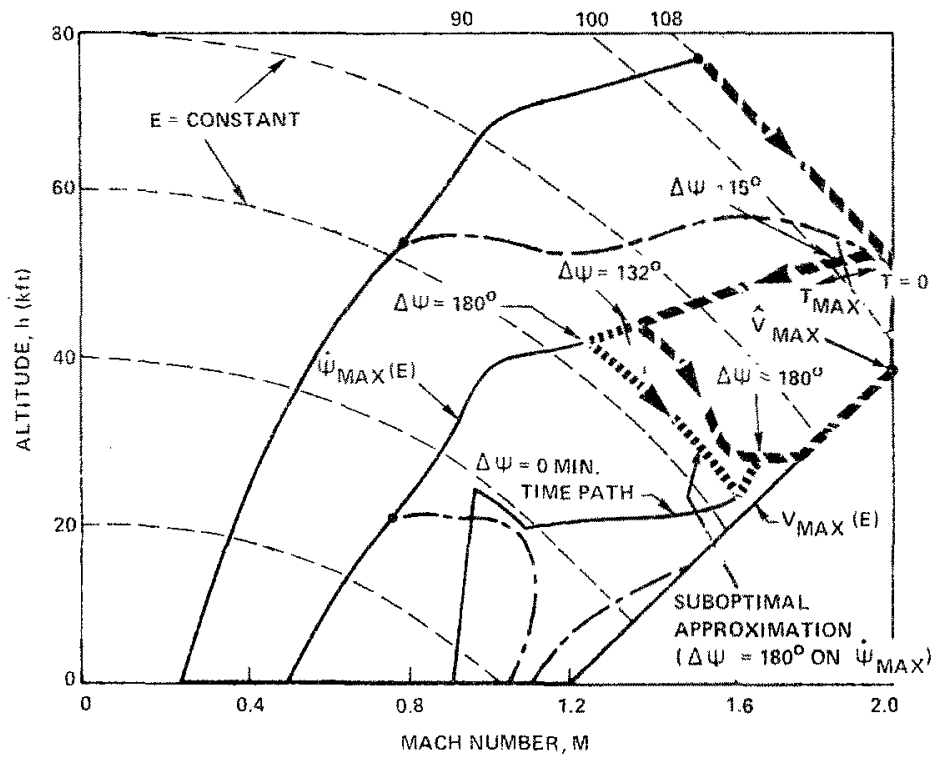

Fig. 11. Minimum-time $180^{\circ}$ turn to a given long range $\left(E_{f}, \psi_{r}\right.$ unspecified), $E_{0}=E_{\max }=108 \mathrm{kft}$.

-DSUBOPTIMAL APPROXIMATHON $(\dot{\Psi}$ MAX TO $\psi=0,200 M$ DIVE TO $\triangle U=0$ MIN. TIME PATHI

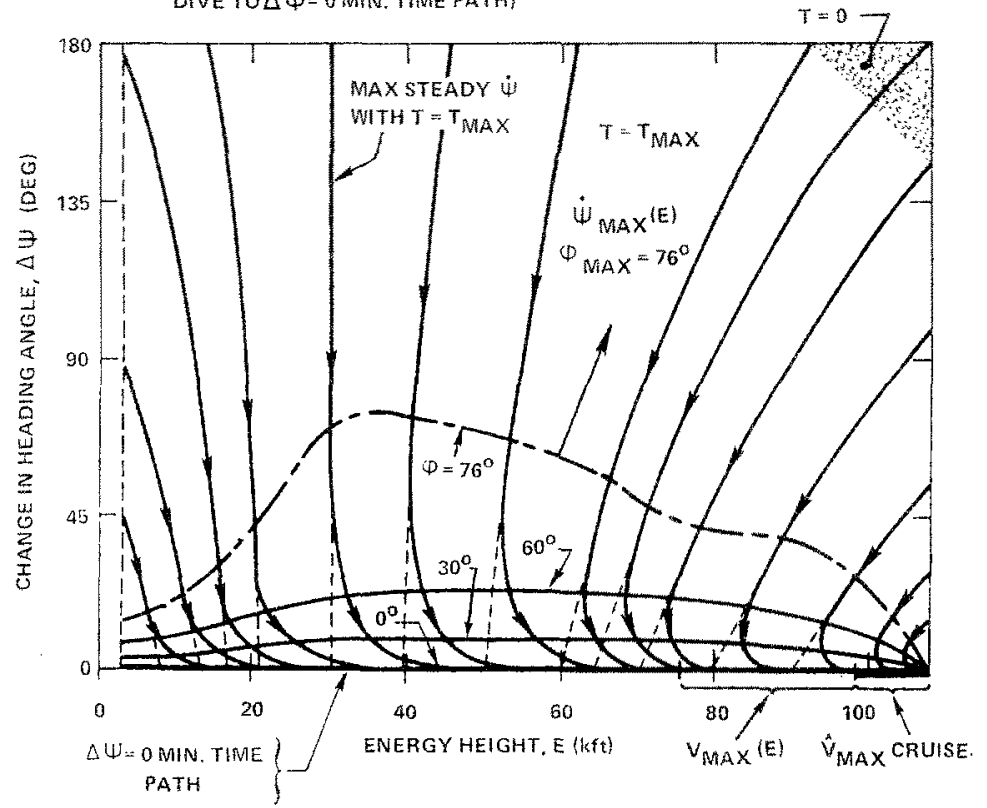

Fig. 12. Minimum-time turns to a given long range $\left(E_{f}, \psi\right.$ unspecified). 


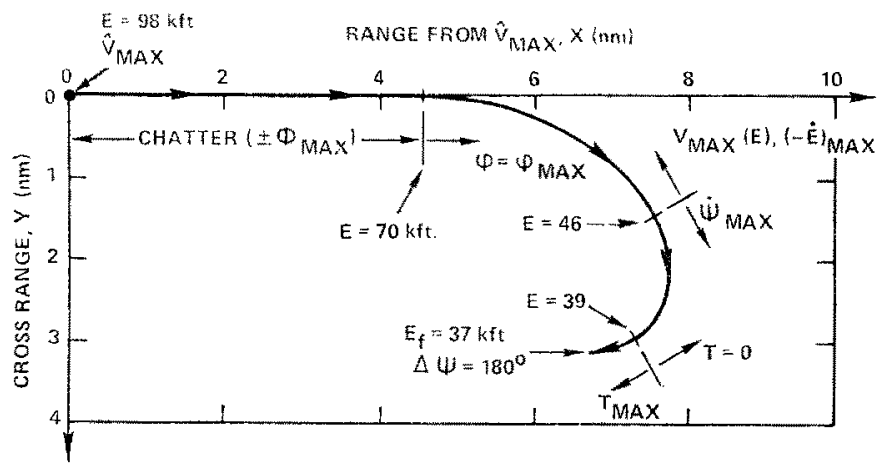

Fig. 13. Horizontal projection of typical minimum-time path to a point from $\hat{V}_{\mathrm{max}}$ cruise.

consists of (a) a straight chatter arc $\left(\phi= \pm \phi_{\max }\right)$ descending in altitude on the $V_{\max }(E)$ boundary with $T=0$ to $E=70 \mathrm{kft}$; (b) a $\phi_{\max }$ turn, still descending on the $V_{\max }(E)$ boundary with $T=0$, to $\Delta \psi \cong 46 \mathrm{kft}$; (c) a zoom climb to the $\dot{\psi}_{\max }$ boundary; (d) a $\dot{\psi}_{\max }$ turn, decelerating with $T=0$, to $\Delta \psi=150^{\circ}, E \cong 39 \mathrm{kft}$; (e) a $\psi_{\max }$ turn decelerating with $T=T_{\max }$, to $\Delta \psi=180^{\circ}, E_{f}=37 \mathrm{kft}$; and finally (f) a zoom climb or dive to the desired final altitude. Note that this same case applies to

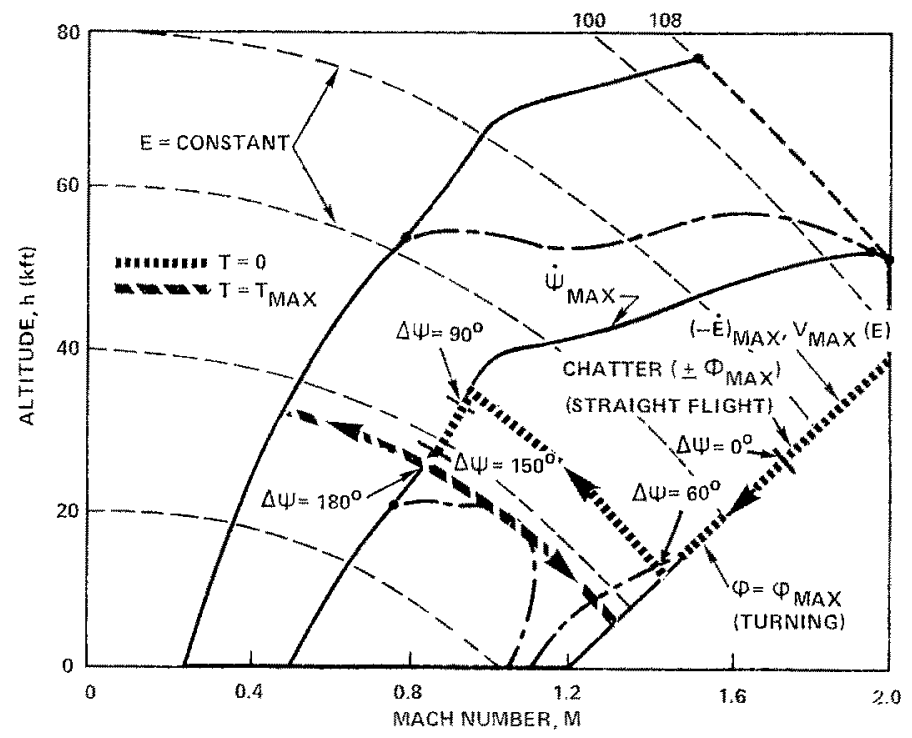

Fig. 14. Minimum-time $180^{\circ}$ turn to a point from $\hat{V}_{\max }$ cruise, $E_{f}=37 \mathrm{kft}$. 


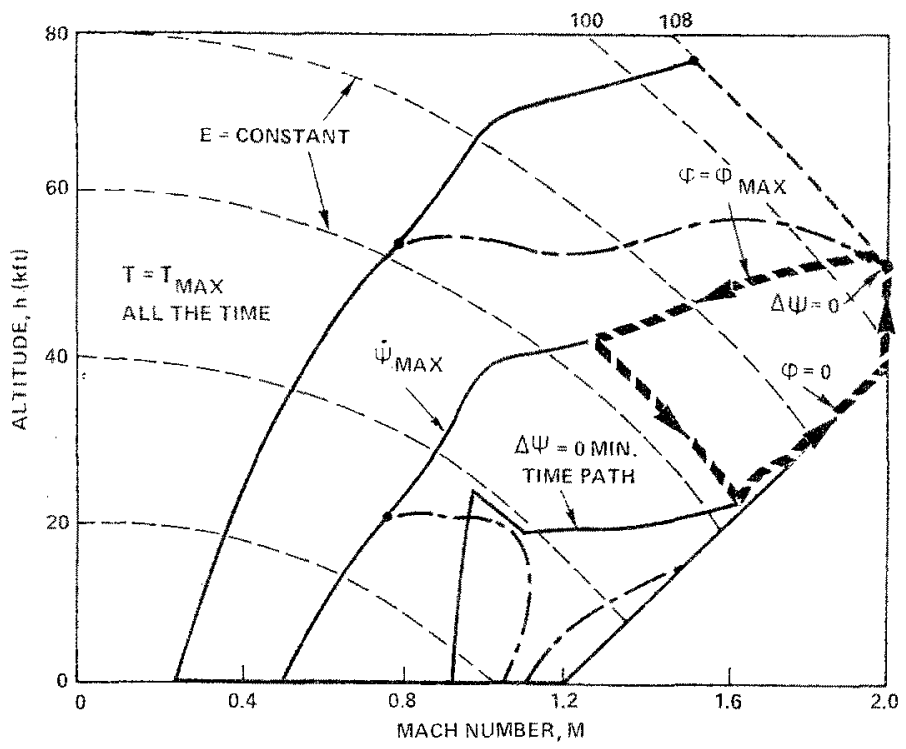

Fig, 15. Winimum-time $180^{\circ}$ turn to a point from $\hat{D}_{\max }$ cruise, $E_{f}=E_{\max }=108 \mathrm{kft}$. turning onto a line $\left(\Delta \psi=\frac{1}{i} 90^{\circ}\right)$ if $E_{f} \approx 44 \mathrm{kft}$ (there would be no $T=T_{\text {max }}$ phase).

Figure 15 shows another type of path for $E_{f}=E_{\max }=108 \mathrm{kft}$, $\Delta \psi=180^{\circ}$ using the suboptimal approximation. It uses $T=T_{\max }$ all the way and consists of four arcs: (a) a $\psi_{\text {max }}$ turn, decreasing energy to $67 \mathrm{kft}$ and turning $180^{\circ}$, (b) a zoom dive to the $\Delta \psi=0$ minimum-time path; (c) a $\phi=0$ segment along the $\Delta \psi=0$ minimum-time path to the $V_{\max }(E)$ boundary, and up that boundary to $M=2$; and (d) a constant Mach number climb at $M=2$ to $E_{f}=108 \mathrm{kft}$.

Figure 16 shows all minimum-time turns to a point or line from $\hat{V}_{\text {mix }}$ cruise with $E_{f}, \Delta \psi_{f}$ as parameters. Note that paths may start anywhere on $\Delta \psi=0,98<E<108 \mathrm{kft}$ assuming that the appropriate energy would have been achieved during cruise by a constant Mach number climb.

\section{Paths that Reach $V_{\max }(\mathrm{E})$ but Not $\ddot{V}_{\max }$}

For all practical purposes, it is also possible to separate arcs for cases where the initial turn reaches $V_{\max }(E)$ but not necessarily $\hat{V}_{\max }$ with $\phi=0 .{ }^{8}$ It is only necessary to match $E_{f}$ of the initial arc to $E_{0}$ of the final arc where, in this case, $75 \leqslant E \leqslant 98 \mathrm{kft}$.

${ }_{8}$ This is true for both the optimal and suboptimal (discrete $\phi$, zoom dives and climbs) cases. 


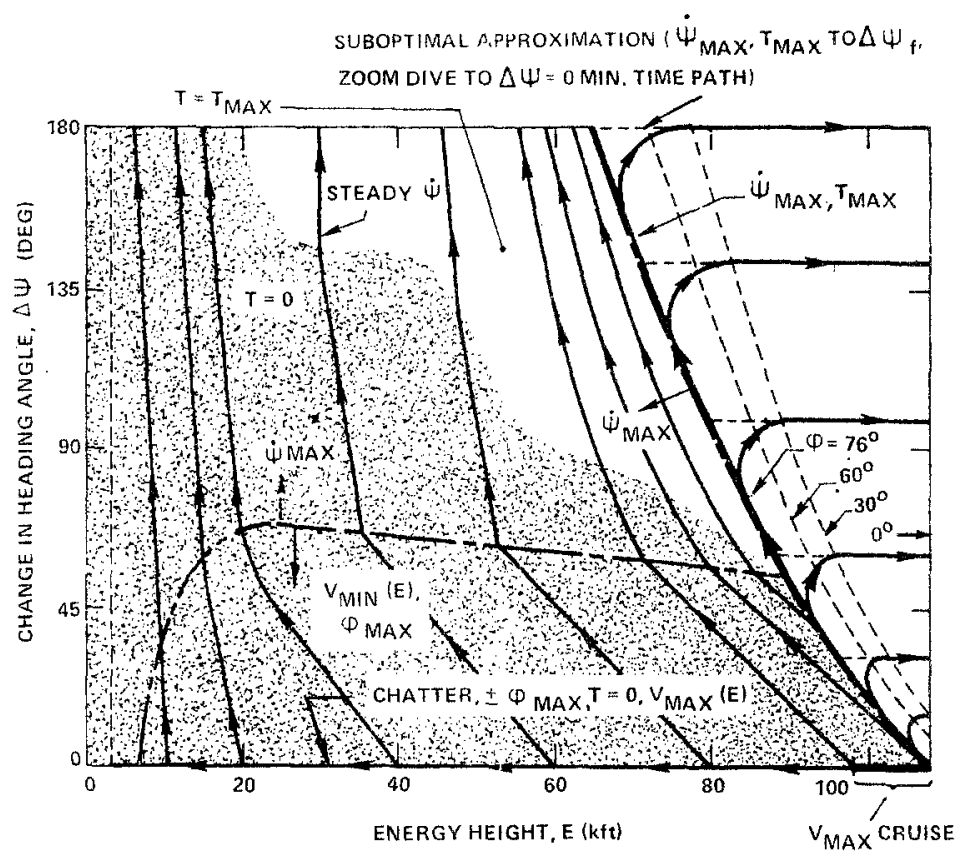

Fig. 16. Minimum-time turns to a point or line from $\hat{V}_{\max }$ cruise.

\section{Conclusions}

When the initial range is sufficiently large that the maximum velocity constraint is encountered en route, the calculation of minimum-time maneuvers can be greatly simplified by a separation of arcs into two two-parameter problems. For shorter ranges, the solution involves five parameters. Suboptimal paths along which the bank angle is restricted to three discrete values $\left(-\phi_{\max }, 0,+\phi_{\max }\right)$ compare favorably with the optimum, continuous bank angle solutions for transonic speeds and below. At very high speeds, the difference becomes more noticeable. However, the suboptimal paths are much simpler to compute and increase the flight time only moderately, thus making them attractive for possible on-line, real-time applications.

\section{References}

1. Bryson, A. E., Jr., Desai, M. N., and Hoffman, W. C., Energy-State Approximation in Performance Optimization of Supersonic Aircraft, Journal of Aircraft, Vol. 6, No. 6, 1969. 
2. Henrick, J. K., and Bryson, A. E., JR., Three-Dimensional, Minimum-Time Turns for a Supersonic Aircraft, Journal of Aircraft, Vol. 9, No. 2, 1972.

3. Parsons, M. G., and Bryson, A. E., JR., Three-Dimensional, Minimum-Time Flight Paths to a Point and onto a Line for a Supersonic Aircraft with a Maximum Mach Number Constraint, Paper No. 32-4, Joint Automatic Control Conference, Stanford University, Stanford, California, 1972.

4. Kelley, H. J., and Lefton, L., Supersonic Aircraft Energy Turns, Paper Presented at the Fifth IFAC Congress, Paris, France, 1972.

5. Parsons, M. G., Three-Dimensional, Minimum-Time Turns to a Point and onto a Line for a Supersonic Aircraft with a Maximum Mach Number Constraint, PhD Dissertation, Department of Applied Mechanics, Stanford University, Stanford, California, 1972.

6. Boyd, J. R., Maximum Maneuver Concept, Informal Briefing, USAF Systems Command Headquarters, Andrews Air Force Base, Maryland, 1971.

7. Patierno, J., Stevens, J. R., and Evanbar, M. S., Application of Optimum $3 D$ Maneuvering Techniques to Parametric Aircraft Design Trade-Offs, USAF FDL Report No. TR-72-57, 1972. 